\title{
Forensic Application of DNA Methylation in Age- Prediction
}

ISSN: 2578-0042

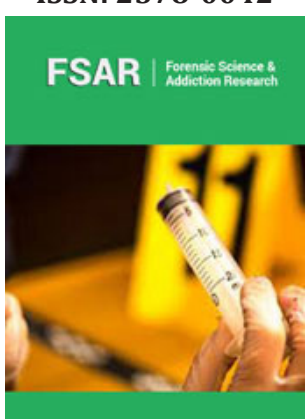

*Corresponding author: Abdurahman Theyab, Department of Laboratory Medicine, Security Forces Hospital, Makkah, Saudi Arabia

Submission: 漹 September 03, 2019 Published: 毕September 12, 2019

Volume 5 - Issue 2

How to cite this article: Abdurahman Theyab. Forensic Application of DNA Methylation in Age-Prediction. Forensic Sci Add Res. 5(2). FSAR.000612.2019. DOI: 10.31031/FSAR.2019.05.000612

Copyright@ Abdurahman Theyab, This article is distributed under the terms of the Creative Commons Attribution 4.0 International License, which permits unrestricted use and redistribution provided that the original author and source are credited.

\author{
Alisraa Althagafi ${ }^{1}$, Mohammad Algahtani ${ }^{2}$ and Abdurahman Theyab ${ }^{3 *}$ \\ ${ }^{1}$ Department of Laboratory Medicine, King Abdulaziz University Hospital, Jeddah, Saudi \\ Arabia \\ ${ }^{2}$ Department of Laboratory Medicine, The Comprehensive Specialized Clinics of The Security \\ Forces, Jeddah, Saudi Arabia
}

${ }^{3}$ Department of Laboratory Medicine, Security Forces Hospital, Makkah, Saudi Arabia

\begin{abstract}
Advances in Epigenetics suggested that DNA methylation profiling, plays an important role in various biological process such as silencing, maintaining or expression of genomic stability. DNA methylation is the most comprehensively studied epigenetic mark, and it involves the addition of a methyl group to the $5^{\prime}$ cytosine of CG dinucleotide, referred to as CpGs. DNA methylation is related to gene regulation and cell differentiation by affecting transcription factor binding sites, insulator components, and chromosome morphology. Recent advances suggested that DNA methylation markers not only can provide information about tissue of origin of evidence sample, but also can rely information about the age and lifestyle or environmental exposure of an individual, for an unknown suspect. In this review, we will be discussing age-associated DNA methylation in various tissues, blood and semen and the applicability of the DNA methylation-based age prediction method to the forensic investigations.
\end{abstract}

\section{Introduction}

DNA methylation defined as the addition of methyl group to the $5^{\prime}$ cytosine of CG dinucleotide (CpGs). As CpG sites across wide range of aging research found to be highly associated with age, multiple age predictive models using DNA methylation at CpG site have been made. DNA methylation considered the most accurate method for age prediction [1]. Variable DNA methylation can be determined by cell differentiation, aging as well as environmental exposure. The established DNA methylation pattern during early years of development are maintained and remain relatively stable throughout life, decrease or increase in different loci [2]. Age-related methylation changes throughout the life of an individual concluded in two phenomena: the epigenetic clock and epigenetic drift. Epigenetic in general is defined as the modification of DNA and DNA packaging without any change in DNA sequence. Epigenetic clock is the age associated DNA methylation changes that are common across individuals of the same age. However, epigenetic drift is the accumulation of small changes or errors that is determined by the environment in which the person ages and it influences $[3,4]$. In epigenetic more specific age-associated DNA methylation changes at certain loci or genes were easier to be detected by the emergence of microarray and massive parallel sequencing technologies (MPS). Although age prediction using DNA methylation is not quite parallel to the chronological age [4]. DNA methylation measures at multiple CpG sites provide close prediction of a chronological age, with a narrow range that varies and specify the accuracy of each method introduced. Thereby, choosing the most appropriate analytical method for forensic application is more difficult than in clinical application. This review will improve the understandings about DNA methylation markers and their potential to be used as biomarkers in the forensic field, with a future plan to apply the previously identified markers on different tissues and test accuracy as well as identify more DNA methylation markers for various tissues, blood and semen.

\section{Tissues}

The age associated CpG sites have been found within a specific tissue and across tissues, because DNA methylation profile are diverse across different type of tissues. Earlier studies, 
by Bocklandt [5] identified three age associated $\mathrm{CpG}$ sites in saliva at the promotor region of EDARADD, TOM1L1 and NPTX2, using the $27 \mathrm{~K}$ Bead Chip array. The regression model resulted and built predicted the age of an individual with an average accuracy of 5.2 years. Following that Koch [6] using five data sets were able to identify 19 CpG hypermethylated aging sites of 20-30 sample each from epidermis, dermis, monocytes, T-lymphocyte and uterine cervical smear using Illumina 27K. Four of these in the GRIA2, TRIM58, KCNQIDN and NPTX2 genes, and a hypomethylation site in the BIRC4BP gene. Age prediction was implemented and the predictive model showed accuracy with mean absolute deviation (MAD) from chronological age of 12.7 years in eight validation sets. Breast tissue repeatedly showed a lower DNA methylation than other cell types at the CG site of the BIRC4BP gene, as it was also described by Horvath [7]. The prediction accuracy increased to 11.4 years of MAD when age prediction was focused on three CG sites in the NPTX2, GRIA2 and KCNQ1DN [8]. Followed his peers path using $450 \mathrm{~K}$ Bead Chip array, was able to demonstrate a high association between the age and DNA methylation in three CpG sites of the genes ELOVL2, FHL2 and PENK in blood. A quantitative model was first built by Hannum [9]. using $71 \mathrm{CpG}$ sites of the $450 \mathrm{~K}$ array and resulted in a very high prediction accuracy of age with an error of $\sim 3.9$ years in blood. Afterward Horvath [7] developed a highly accurate model, that can be implemented on multiple tissue for age prediction by allowing an accurate estimation of DNA methylation age of multiple cells and tissues. The analysis results of 7,840 non-cancer samples from 82 data sets produced using Illumina 27K or Illumina's Human Methylation450 Bead Chip array (Illumina 450K), which encompass 51 different tissues and cell types. The 353CpGs that were automatically selected from the elastic net allowed highly accurate age prediction with an error of 3.6 years in the test data.

\section{Blood based samples}

Blood was also used in DNA methylation age-prediction. Pyrosequencing of blood at three CpGs at the genes ASPA, PDE4C and ITGA2B for age prediction was described by Weidner [10]. The model demonstrated high accuracy with a mean absolute deviation (MAD) from chronological age of 4.3 years. This was followed by Piekarska [11] who reported an age predictive model for blood using two CpGs in the ELOVL2 gene. Zbiec model had a prediction error of 6.85 years and accuracy of 5.03 years MAD from chronological age. Another model of five CpG sites of the genes TRIM59, C1orf132, FHL2, ELOVL2 and KLF14 was established and had an accuracy with a mean absolute deviation (MAD) from chronological age of 3.9 years [12]. Lately Park [13] reported an age predictive model for blood using three CpG site of the genes CCDC102B, ZNF423, and ELOVL2 by evaluating of 760 blood samples based on a pyrosequencing platform. The model was highly accurate with a MAD from the chronological age of 3.4 years. Of all the genes tested, DNA methylation at FHL2 and KLF14 were significantly associated with age; yet, the primer design for pyrosequencing of these genes failed. As a consequence of the high association between the age and DNA methylation at the genes FHL2, KLF14, and ELOVL2, it has been repeatedly observed in many studies with blood, they are considered the most promising age-predictive markers for blood.

\section{Semen}

Lately, Lee [14] identified a new age associated CpG sites found in semen samples, using the $450 \mathrm{~K}$ array and subsequent methylation Snapshot analyses. Semen is a particularly important body fluid in the forensic analyses. Although, Horvath model (we discussed earlier) is applicable for age-prediction in several types of cells and tissues, the age prediction values for sperm were much lower than the chronological age of the donors [7]. The model by Lee et al. showed a high correlation between the chronological and the predicted age and it consisted of three CpG sites (cg12837463, cg06979108 in the NOX4 gene, and cg06304190 in the TTC7B gene) a MAD from chronological age of $\sim 5$ years. Furthermore, the area around the TTC7B gene showed an alteration in DNA methylation in the sperm methylome of two samples collected 9-19 years apart from each individual [15]. Thus, indicating TTC7B as one of the most promising age predictive marker for semen. Also, Bekaert et al. [16] developed a model with a four age-associated markers suggested for blood PED4C, EDARADD, ELOVL2 and ASPA. The model proved to be highly accurate for age predictions using teeth samples with a MAD from chronological age of 4.9 years. Giuliani [17] also proved that the previously reported age-associated markers for blood, i. e., CpG sites located in the PENK, ELOVL2 and FHL2 genes [8], could be a great tool to predict the age in teeth; although, the mean absolute deviation (MAD) from chronological age, depending on the part of the tooth from which the sample taken, varied between 1.2-7.1 years.

\section{Conclusion}

DNA methylation and age-prediction models can be applied across broad spectrum of tissues, but the accuracy varies depending on the tissue type. In the field of forensics, the prediction accuracy of a model considered high if the MAD is less than 5 years, models for age prediction in blood, saliva, semen have been reported using only a few age-associated CpGs, that measure the biological age and provide information about life expectancy as well as unknown sample donor's appearance [18]. We believe, in the future, DNA methylation markers will play an important role in the forensic investigations as well as in also in clinical research.

\section{References}

1. Schübeler D (2015) Function and information content of DNA methylation. Nature 517(7534): 321-326.

2. Lee HY, Lee SD, Shin KJ (2016) Forensic DNA methylation profiling from evidence material for investigative leads. BMB Rep 49(7): 359-369.

3. Jones MJ, Goodman SJ, Kobor MS (2015) DNA methylation and healthy human aging. Aging Cell 14(6): 924-932.

4. Jung SE, Shin KJ, Lee HY (2017) DNA methylation-based age prediction from various tissues and body fluids. BMB Rep 50(11): 546-553.

5. Bocklandt S, Lin W, Sehl ME, Sánchez FJ, Sinsheimer JS, et al. (2011) Epigenetic predictor of age. PLoS One 6(6): e14821.

6. Koch CM, Wagner W (2011) Epigenetic-aging-signature to determine age in different tissues. Aging (Albany NY) 3(10): 1018-1027. 
7. Horvath S (2013) DNA methylation age of human tissues and cell types. Genome Biol 14(10): R115.

8. Garagnani P, Bacalini MG, Pirazzini C, Gori D, Giuliani C, et al. (2012) Methylation of ELOVL2 gene as a new epigenetic marker of age. Aging Cell 11(6): 1132-1134.

9. Hannum G, Guinney J, Zhao L, Zhang L, Hughes G, et al. (2013) Genomewide methylation profiles reveal quantitative views of human aging rates. Mol Cell 49(2): 359-367.

10. Weidner CI, Lin Q, Koch CM, Eisele L, Beier F, et al. (2014) Aging of blood can be tracked by DNA methylation changes at just three CpG sites. Genome Biol 15(2): R24.

11. Piekarska Z, Spólnicka R, Kupiec M, Makowska T, Spas Z, et al. (2015) Examination of DNA methylation status of the ELOVL2 marker may be useful for human age prediction in forensic science. Forensic Sci Int Genet 14: 161-167.

12. Piekarska Z, Spólnicka R, Kupiec M, Proszek TP, Makowska A, et al (2015b) Development of a forensically useful age prediction method based on DNA methylation analysis. Forensic Sci Int Genet 17: 173-179.
13. Park JL, Kim JH, Seo E, Bae DH, Kim SY, et al. (2016) Identification and evaluation of age-correlated DNA methylation markers for forensic use. Forensic Sci Int Genet 23: 64-70.

14. Lee HY, Jung SE, Oh YN, Choi A, Yang WI, et al. (2015) Epigenetic age signatures in the forensically relevant body fluid of semen: A preliminary study. Forensic Sci Int Genet 19: 28-34.

15. Jenkins TG, Aston KI, Pflueger C, Cairns BR, Carrell DT (2014) Ageassociated sperm DNA methylation alterations: Possible implications in offspring disease susceptibility. PLoS Genet 10(7): e1004458.

16. Bekaert B, Kamalandua A, Zapico SC, Van de Voorde W, Decorte R (2015) Improved age determination of blood and teeth samples using a selected set of DNA methylation markers. Epigenetics 10(10): 922-930.

17. Giuliani C, Cilli E, Bacalini MG, Pirazzini C, Sazzini M, et al. (2016) Inferring chronological age from DNA methylation patterns of human teeth. Am J Phys Anthropol 159(4): 585-595.

18. Jones PA (2012) Functions of DNA methylation: Islands, start sites, gene bodies and beyond. Nat Rev Genet 13(7): 484-492.

For possible submissions Click below: 